\title{
INTERVIEW
}

For reprint orders, please contact: reprints@futuremedicine.com

\section{One stop multidisciplinary pain clinic for survivors of torture}

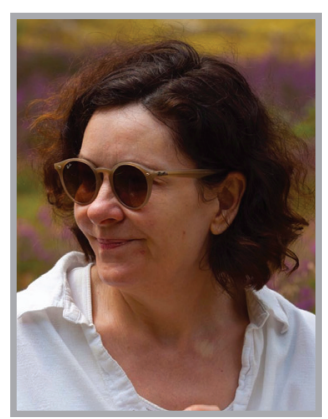

Bianca Kuehler,1 and Susan Childs ${ }^{*, 1}$ speak to Jade Parker, Commissioning Editor:

Dr Bianca Kuehler initially qualified in Germany as an anesthetist in 1993 and is on the specialist register in the UK. After moving to the UK she obtained a Diploma in Occupational Health to supplement the understanding and implication of chronic pain on the work environment. She is very interested in multidisciplinary approaches in treatment of chronic and acute pain patients and, therefore, working closely with $\mathrm{Dr}$ Childs opened a plethora of opportunities to develop new services including a fibromyalgia clinic and a specialist clinic for patients who are survivors of torture.

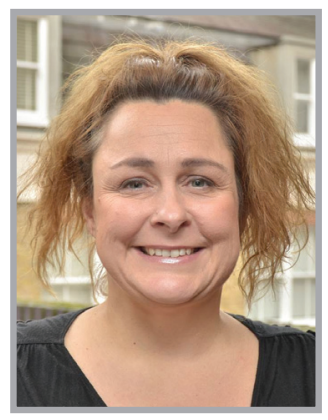

Dr Susan Childs is an experienced clinical psychologist who has worked within health psychology and mental health since 1997. Her particular area of expertise is chronic pain. Alongside this, she has developed expertise in the assessment and treatment of a wide range of psychological issues. She has more recently focused upon co-developing services alongside her medical lead and co-facilitator, $\mathrm{Dr}$ Bianca Kuehler, for patients who are survivors of torture. Susan leads therapy services at a major London National Health Service trust in a Consultant capacity and supports a team of physicians, surgeons, pain specialist physicians, physiotherapists and clinical specialist nurses.

First draft submitted: 16 June 2016; Accepted for publication: 13 July 2016; Published online: 19 August 2016

Q You are the joint lead clinicians for the 'One stop multidisciplinary pain clinic for survivors of torture'. What are the aims of the clinic \& how are you managing or treating these patients differently?

- BM Kuehler

The aims are offering a service to a vulnerable group of patients that is tailored to their specific needs taking into account that they should receive the most appropriate evidence-based treatment available. As their needs are both physical and psychological a one-dimensional approach would not be able to cover this. In our clinic we combine a specialist pain clinic appointment with a medication review clinic and a psychology assessment in one set-up.

Q What made you first consider the needs of patients who have survived

\section{KEYWORDS}

- pain assessment $\bullet$ pain management $\bullet$ post-traumatic pain

'Chelsea \& Westminster NHS Foundation Trust, 369 Fulham Rd, London, SW10 9NH, UK

*Author for correspondence: bianca.kuehler@chelwest.nhs.uk

**Author for correspondence: susan.childs@chelwest.nhs.uk

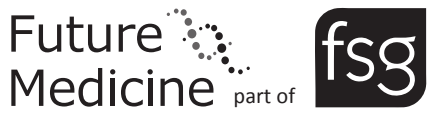


torture as being different from a routine pain clinic?

\section{- BM Kuehler}

This group of patients have undergone both physical and psychological severe trauma. The trauma was inflicted by another human being and even healthcare professionals including doctors might have been involved. Building trust is paramount. We found that these patients were struggling with the routine set-up of the pain clinic. There was not enough time to identify issues and address the trauma, build trust and develop a treatment plan in the normal appointment time. Additionally by talking about their experience there is an increased risk of psychological dissociation and as medical doctors we are not ideally placed to deal with this. We simply needed something different and more specific.

\section{- SR Childs}

Our survivor of torture (SoT) patients clearly struggled to even wait in the general clinic area and we began to notice that on clinic days with heavy footfall that our SoT patients became distressed by the busy, noisy environment. Many of the patients also became increasingly upset by the constant news channel that played in the waiting area. Thanks to the CW+ charity, this has now been changed to a $3 \mathrm{D}$ picture that shows calming, nonpolitical images.

\section{- BM Kuehler}

A huge problem that we noticed was that this group of patients would not attend psychology appointments, as though they feared that their pain and suffering would be explained away by mental illness. We also noticed an increase in the number of times I needed to ask Dr Childs to attend my sessions with SoT as they presented with uncontrolled psychological illness or symptoms. Therefore, we decided that the easiest way forward is to offer them a combined appointment with both of us.

Now, once a patient has been identified as being a SoT they can be directly booked into our clinic. Everyone in our team has this option. Our team is now comfortable to address issues of previous exposure to human cruelty after we organized a training day with the 'Freedom from Torture' charity to teach us how to communicate more effectively with these patients.

\section{- SR Childs}

The Chelsea and Westminster Hospital
Pain Services has a long history of offering population-specific services. We began by offering specialist pain or intervention clinics for Arabic speaking women patients who displayed a low level of attendance in pain management programs. From there the team looked at patients who were considering spinal cord stimulators, which was then followed by a one-stop clinic model for patients with fibromyalgia. We also have a specialist neuropathic pain service. All of these clinics offered specific pain management solutions for patient groups who did not fit into the usual clinics we offered. The Survivors of Torture Pain Clinic came about after we identified that these patients, more than any other group, did not fit in the 'one-size-fits-all model'.

Q Are there any particular pain management success stories or events that have particular meaning to you in relation to the SoT clinic?

\section{- SR Childs}

We gave a talk at a hospital governors' meeting which was open to the public about the Survivors of Torture Clinic and its aims. In the audience was a face I recognized who was smiling and clapping. At the end, the gentleman came to talk to me and said that he was one of the first patients who came through the clinic when we started it. He told us that he had gone into one of our pain management programs (which is unusual as they often struggle in our mainstream groups) and from there managed to get a job as a hotel manager and had only stopped working when he had sadly experienced a heart attack. However, he said that he was still using the strategies we had taught him to manage his pain and was working his way back to the things that he valued. It was so lovely to see someone who had really managed to move toward his life values using pain management strategies.

\section{- BM Kuehler}

One patient was telling us that he did not want further investigations with needles or injections. He clearly had nerve damage from the torture he had experienced and when he saw a doctor, further tests were organized. Every time this happened his trauma and pain were exacerbated. We were able to write an extensive report to clarify that the patient would not benefit from further investigations as long as his condition was stable. When we saw him again he was much happier 
and was able to engage with pain management strategies.

\section{Q How big is the issue of undertreatment of pain in SoT?}

\section{- BM Kuehler}

This is a big problem. Undertreatment can involve many areas, the biggest problem being unable to find a psychologist to look at pain management strategies with a patient. Another problem is noncompliance with medications as the patients depending on their cultural background might have a different understanding of use. We found some patients hoarding drugs so a cache was available in case the pain might get worse or perscriptions changed. Also taking medications such as antineuropathic medications can be difficult as they do not immediately see the benefit. In our clinic we do extensive education about appropriate medication use as we are able to offer a nurse-led medications review clinic as part of the SoT clinic to discuss their medications and their effects.

Another problem is that SoT often do not attend interventional treatments as they are afraid of coming to an operating theater. But often we see the contrary as well; patients being overtreated without any benefit. We see many of them after failed interventions, including injections and even surgery.

\section{Q Why is it important to have a} multidisciplinary team structure in this clinic?

\section{- SR Childs}

The multidisciplinary team (MDT) structure of the team is in our opinion essential in managing these patients well. It offers SoT the chance to meet with a consistent team of clinicians; they have access to a general medications review and a dual medical and psychological assessment.

The dual assessment is carried out by the two lead clinicians and this truly takes into account both the body and the mind. It also allows the psychologist to be introduced to each patient and time to discuss how and what they may be offered as treatments (both medical and psychological) and then to be given dates for the same. Patients are followed up in this clinic (i.e., scheduled on the quietest day in terms of foot fall in the clinic area).

Q You \& your team recently won the Grünenthal Pain Award, how do you feel about winning \& what will the award mean for the clinic?

- SR Childs

We are over the moon at having won this award. It is incredibly difficult to publicize services in the National Health Service (NHS). We have tried to approach newspapers and magazines but have been unsuccessful. This award will help us to raise our profile and hopefully lead to helping other services think about changing how they manage their population of these vulnerable and often unheard or unseen patients.

The prize money from the Grünenthal awards is going to allow us to increase our MDT power. We will look to buy some psychiatrist hours so that those who really do need a psychiatric assessment or a MDT discussion can be seen without relying on having to ask the GP to refer (this also helps reduce the patient having to recall traumatic memories/their torture story again to a doctor they may have not disclosed to in an environment that perhaps they have not considered safe in the past).

\section{- BM Kuehler}

The award gives us more visibility and this was one of our major goals. We want to make our colleagues both in the pain community and surgeons aware that these patients are more complex and need a different approach. It gives us a voice for the unheard and under-represented torture survivors. This will also enable us to collect and publish our data much more easily.

\section{Q How does the medical management for} these patients differ?

\section{- BM Kuehler}

Identification is the key. We find that there are two groups of patients, one that has been obviously tortured and presents with symptoms directly relating to the torture. The other group consitsts of the patients who initially did not have ongoing chronic pain after torture, but present with severe pain after developing another pain, for example low back pain. They might present with significant pain behaviors. If the pain is then not understood in context of previous torture, these patients might be massively overtreated or dismissed. Pain for this group of patients is most likely experienced as threat and a constant reminder of the torture, maybe not consciously but subconsciously. If this aspect is not addressed or left too late, they can become 
severely deconditioned and stop participating in normal activities of daily living. They also would not respond to usual treatment.

We spend a lot of time looking at their medications and explaining how to use them properly. Sarah Thomas, CNS, our lead nurse in Pain Management, is seeing the patients first to educate them about their medications. This gives them time to relax as they talk about something familiar and nonthreatening. In the dual session with Dr Childs, I will establish any medical needs or investigations required. If appropriate we can then also discuss interventions. We have time to explain exactly what is going to happen. We can then look at pain management strategies together. By doing this with me as a clinician I am validating these treatments and the patients are more willing to engage with them. We find that this is an essential part in the treatment as the misunderstanding of what modern medicine can do and the limitations of this is often huge.

\section{Q You described in your book that you use cognitive-behavioral therapy for persistent pain presentations \& co-morbid issues. Is this the best model for SoT? \\ - SR Childs}

The cognitive-behavioral therapy model is difficult to apply to our patients who have survived torture. Many of the techniques used could allow flashbacks associated with trauma to come back into the patients' focus and to challenge the thoughts about being tortured with commonly used questions in cognitivebehavioral therapy ('is it true' 'is it useful') is not helpful as the thoughts are often horribly true. Instead we try to focus on what is useful to each patient and what they identify as being activities that they value that can bring them closer to a wider life where pain is not the sole focus. This often involves another member of our MDT - physiotherapists. We introduce them with the idea that we are going to try and build up their bodies and their minds. These sessions often involve exercises, for example, to help them play with their children and are lovely when they report that they feel closer to them as a consequence of the sessions that follow the SoT pain clinic. In the future we would like to explore a more compassionate, focused model to see if helping these patients get in touch with a sense of compassion for themselves could be helpful (we know that it is proven with trauma patients but to our knowledge has not been explored with SoT).

\section{Q You are the co-author of 'The Practical Pain} Management Handbook', how would you like to see the field of pain management develop in the next few years?

\section{- SR Childs}

First, I would very much like to see more interconnection between services available within the NHS. Many trusts have pockets of excellence/ specialty services but we as tertiary clinicians can only access them through referring back to the GP, even then acceptance into these services is not guaranteed. If we could refer from our specialist clinic to another that would help the patient a great deal and perhaps allow that service to fund and develop these 'unseen specialties'. I would also like to see some of the pain management services delivered in a space more consistent with a self-management message, such as local gyms, as this would help people identify and adhere to the strategies taught. Finally, I would like the support group that runs off the back of the Chelsea \& Westminster to gain a wider publicity as it could offer ongoing support for people with little social networks as I think with dwindling financial support for the NHS these expert patient groups could develop a strong supportive role.

\section{Q Do you have any closing comments or messages for our readers? \\ - BM Kuehler}

To be aware that often healthcare professionals took part in the torture and possibly even wellmeaning treatments, and that our interaction with the patients can lead to re-traumatization. To also consider that a patient might have experienced torture in the past even when they report onset of pain more recently, especially when the pain is reported as severe and affects their day to day activities. This group gets easily overlooked and treatment then can be inappropriate.

\section{- SR Childs}

Throughout history, people have had to move away from conflict, deprivation and maltreatment. In the UK we have a rich and varied cultural population mix. An increasing part of this population comes to our country having been tortured. This changes how they view the world and how they tolerate medical/therapeutic treatment. The Survivors of Torture Clinic at the Chelsea and Westminster Hospital did not 
start out with any specific financial support. We adapted the existing service to meet this population's needs with little financial impact. This is a 21 st century, low-cost solution to a 21st century UK population need.

\section{Disclaimer}

The opinions expressed in this interview are those of the interviewees and do not necessarily reflect the views of Future Medicine Ltd.
Financial \& competing interests disclosure

BM Kuehler and SR Childs have previously received speaker's fees from Grünenthal. BM Kuehler received speaker's fees from Pfizer. BM Kuehler and SR Childs have no other relevant affiliations or financial involvement with any organization or entity with a financial interest in or financial conflict with the subject matter or materials discussed in the manuscript apart from those disclosed.

This interview has been conducted with editorial support from Lynsey Conway, funded by Grünenthal. 